\title{
Best conditions for biodegradation of diesel oil by chemometric tools
}

\author{
Ewa Kaczorek ${ }^{1}$, Katarzyna Bielicka-Daszkiewicz ${ }^{1}$, Károly Héberger ${ }^{2}$, Sándor Kemény ${ }^{3}$, \\ Andrzej Olszanowski ${ }^{1}$, Adam Voelkel ${ }^{1}$ \\ ${ }^{1}$ Institute of Chemical Technology and Engineering, Poznan University of Technology, Poznan, Poland. \\ ${ }^{2}$ Chemical Research Center, Hungarian Academy of Sciences, Budapest, Hungary. \\ ${ }^{3}$ Department of Chemical and Environmental Process Engineering, \\ Budapest University of Technology and Economics, Budapest, Hungary.
}

Submitted: January 16, 2012; Approved: April 1, 2013.

\begin{abstract}
Diesel oil biodegradation by different bacteria-yeast-rhamnolipids consortia was tested. Chromatographic analysis of post-biodegradation residue was completed with chemometric tools (ANOVA, and a novel ranking procedure based on the sum of ranking differences). These tools were used in the selection of the most effective systems. The best results of aliphatic fractions of diesel oil biodegradation were observed for a yeast consortia with Aeromonas hydrophila KR4. For these systems the positive effect of rhamnolipids on hydrocarbon biodegradation was observed. However, rhamnolipids addition did not always have a positive influence on the biodegradation process (e.g. in case of yeast consortia with Stenotrophomonas maltophila KR7). Moreover, particular differences in the degradation pattern were observed for lower and higher alkanes than in the case with C22. Normally, the best conditions for "lower" alkanes are Aeromonas hydrophila KR4 + emulsifier independently from yeasts and e.g. Pseudomonas stutzeri KR7 for $\mathrm{C} 24$ alkane.
\end{abstract}

Key words: biodegradation, chemometrics, systems ranking, variance analysis.

\section{Introduction}

Petroleum is a complex mixture of non-aqueous and hydrophobic compounds such as alkanes, aromatics and asphaltenes. The low accessibility of hydrophobic compounds to microbial cells causes their slow biodegradation. Addition of surfactants enhances the efficiency of the removal of organic contaminations by increasing their bioavailability (Aronstein and Alexander, 1993; Liu et al., 1995; Volkering et al., 1995). Different surfactants were used in the bioremediation process. However, biosurfactants represent ecological alternatives to their synthetic counterparts: exhibiting lower toxicity, potentially high activities and stabilities at extreme temperatures and $\mathrm{pH}$ (Abdel-Mawgoud et al., 2010). However, rhamnolipids sometimes have antimicrobial properties used against bacterial and fungal species (Benincasa et al., 2004; Haba et al., 2003). Various bacterium strains could be able to degrade crude oil either in single or mixed form (Kaczorek and Olszanowski, 2011; Palittapongarnpim et al., 1998) and also bacteria and a yeast consortium (Horakova and Nemec, 2000).

A number of biodegradation data of complex systems requires the use of various chemometric methods/techniques to select the optimal - most effective system for the biodegradation of diesel oil. Modeling biodegradation is very difficult, because petroleum is a complex mixture. The lipophilicity and the solubility of its organic compounds change as the molecular mass increases. Diesel oil biodegradation is associated with concentration changes of C11-C24 alkanes. A full factorial experimental design displays the influence of various factors on the degradation process, namely: quality of bacterial strains, effect of yeast and presence or absence of emulsifiers. Variance analysis (ANOVA) is a suitable tool for distinguishing the effects of different factors. The results were compared to the ordering systems using a novel ranking method based on the sum of ranking differences. 
The aim of this investigation was to examine the influence of the selected rhamnolipids onto a yeast/bacterial consortium bioactivity during the biodegradation process of diesel oil. Special attention was paid to the biodegradation possibility of some fractions of biodegraded oil.

\section{Material and Methods}

\section{Growth conditions of microorganisms}

Three bacterial strains were isolated from crude oil contaminated soil: Aeromonas hydrophila KR4, Stenotrophomonas maltophila KR7 and Pseudomonas stutzeri KR7 were used in the experiments and denoted by $A, X$ and $P$, respectively. A soil sample was introduced into the culture medium solution containing appropriate amounts of nitrogen and phosphorus as well as trace elements. In order to amplify microorganisms in the initial phase glucose as a source of carbon was introduced. After $24 \mathrm{~h}$ of cultivation, microorganisms were transferred into the new culture medium system, containing diesel oil as a carbon source. The cultivation then was carried out for a month with microorganisms transferred every $48 \mathrm{~h}$ into a new medium with increasing amounts of diesel oil. This allowed for the isolation of bacterial strains with the potential ability of biodegradation. The identification of the bacterial strain was performed using biochemical tests ID 32 GN (prod. bio-Merieux, France) and molecular techniques. The yeast strains: Candida maltosa EH15 - 1 and EH60 - 2 as well as Yarrowia lipolytica EH59 - 3 and EH425 - 4 were used in experiments (Chrzanowski et al., 2008) - their notations (numerations) are given in bold.

The culture medium used throughout the studies consisted of (g/L): $\mathrm{Na}_{2} \mathrm{HPO}_{4} \cdot 2 \mathrm{H}_{2} \mathrm{O}$ 7.0, $\mathrm{KH}_{2} \mathrm{PO}_{4} 2.8, \mathrm{NaCl}$ $0.5, \mathrm{NH}_{4} \mathrm{Cl} 1.0, \mathrm{MgSO}_{4} \cdot 7 \mathrm{H}_{2} \mathrm{O} 0.01, \mathrm{FeSO}_{4} \cdot 7 \mathrm{H}_{2} \mathrm{O} 0.001$, $\mathrm{MnSO}_{4} \cdot 4 \mathrm{H}_{2} \mathrm{O} \quad 0.0005, \mathrm{ZnCl}_{2} 0.00064, \mathrm{CaCl}_{2} \cdot 6 \mathrm{H}_{2} \mathrm{O}$ $0.0001, \mathrm{BaCl}_{2}$ 0.00006, $\mathrm{CoSO}_{4} 7 \mathrm{H}_{2} \mathrm{O} 0.000036, \mathrm{CuSO}_{4} \cdot$ $5 \mathrm{H}_{2} \mathrm{O} 0.000036, \mathrm{H}_{3} \mathrm{BO}_{3} 0.00065$, EDTA 0.001 , and $\mathrm{HCl}$ $37 \% 0.0146 \mathrm{~mL}$. The $\mathrm{pH}$ of the medium was 7.2. Yeast extract $(0.3 \mathrm{~g} / \mathrm{L})$ was added to the bacteria stock cultures. Stock cultures (bacteria and yeast) were prepared in a $250 \mathrm{~mL}$ Erlenmeyer flask containing $50 \mathrm{~mL}$ of medium. Next, a loop full of cells from an agar plate was added to the flask with the medium. After approximately $24 \mathrm{~h} 3-5 \mathrm{~mL}$ of this liquid culture was used for the inoculation of the final culture to reach an OD of ca. 0.1 (corresponds to $1.10^{8}$ cells per $\mathrm{mL}$ ).

For single bacterial strains and yeast strains, twelve consortia were prepared. Each of the three tested strains: $A$. hydrophila KR4, S. maltophila KR7, P. stutzeri KR7 was mixed with each of the four yeast strains: C. maltosa EH15, C. maltosa EH60, Y. lipolytica EH56, Y. lipolytica H465. The inoculation of the final culture was performed in such a way that the initial concentrations of both bacteria and yeast were equal to OD 0.1 respectively. This corresponds to $1.10^{8}$ cells per $\mathrm{mL}$ of bacteria and also to $1.10^{8}$ cells per
$\mathrm{mL}$ of yeast. The final bacterium to yeast ratio was assessed counting CFU after transfer on agar plates. The bacterium to yeast ratio at the end of the experiment was ca. 40:60.

Microbial growth was monitored through culture densities, measuring absorption spectrophotometrically at $600 \mathrm{~nm}$ (data not given).

\section{Chemicals}

Hydrocarbons and other fine chemicals employed in the study were of the highest purity grade, produced by Merck (Germany). Crude Oil Quantitative Standard was from Supelco. Surface active agents used in the experiments were rhamnolipids (Jeneil Biosurfactant Company, USA, JBR 425 - content $25 \%$ of rhamnolipids).

\section{Biodegradation test}

Diesel oil was used as a carbon source for microorganism biodegradation. Hydrocarbon concentration used in the experiments was $2 \%(\mathrm{w} / \mathrm{v})$. The influence of rhamnolipids biosurfactant on diesel oil biodegradation was also tested. Surfactants were used at $120 \mathrm{mg} / \mathrm{L}$ concentrations. Their presence is denoted by the letter "E"; i.e. AE2 means a combination of $A$. hydrophila KR4, with yeast strain: C. maltosa EH60 - 2 in the presence of ramnolipids. Altogether 24 systems are defined (denoted by A1, A2, A3, A4, AE1, ..., X4, $\mathrm{XE1}, \ldots, \mathrm{PE} 3, \mathrm{PE} 4)$. Laboratory tests with different surfactant concentrations showed that diesel oil biodegradation in the presence of such an amount of surfactant was the most effective. Experiments were performed in Erlenmeyer flasks containing $50 \mathrm{~mL}$ of culture medium. Experiment samples contained: diesel oil, a culture medium and a few $\mathrm{ml}$ of bacteria stock cultures (to reach an OD of ca. $0.1)$. In the case of experiments with emulsified hydrocarbon, an appropriate amount of surfactants were added to the prepared samples. Each experiment was repeated five times, and the values of the biodegradation were calculated as a mean value of five flasks to attain an accuracy of $\pm 3.2 \%$. Samples were incubated at $25^{\circ} \mathrm{C}$ and shaken at $120 \mathrm{rpm}$ for 7 days. The effect of the three factors (bacteria, yeast and presence or absence of emulsifier) on diesel oil biodegradation was tested. After the biodegradation process, the whole cultivation broth was centrifuged in order to separate biomass. Saturated salt solution and acids were added to the residual aqueous phase to achieve a $\mathrm{pH}$ of 1.0. The aqueous phase was then double extracted with diethyl ether. The organic phase after extraction was dried and then evaporated. One of the dried residues was dissolved in ethyl acetate and measured using gas chromatography.

\section{GC experiments}

Gas chromatography was used to determine aliphatic hydrocarbon content in crude diesel oil and in the residues after the biodegradation process. Qualitative and quantitative analyses were carried out, on the HP 5890II gas chromatograph with flame ionization detector (GC-FID) 
equipped with autosampler. Other details of GC experiments were as follows: capillary column 50\% cyanopropylmethyl, 50\% phenylmethyl polisiloxane DB-225 (Agilent Technologies) $30 \mathrm{~m}$ x $0.25 \mathrm{~mm}$ I.D., film thickness $0.25 \mu \mathrm{m}$. Helium was used as a carrier gas at a flow-rate of $1.5 \mathrm{~mL} / \mathrm{min}$ and head pressure of $90 \mathrm{kPa}$. The injector and detector temperatures were $300{ }^{\circ} \mathrm{C}$. The column temperature was held at $60{ }^{\circ} \mathrm{C}$ for $1 \mathrm{~min}$, and then ramped at $10^{\circ} \mathrm{C} / \mathrm{min}$ to $220^{\circ} \mathrm{C}$ where it was held for $10.5 \mathrm{~min}$. The injection volume was $5 \mu \mathrm{L}$.

For qualitative and quantitative determination of crude diesel oil analysis of model samples was done. The mixture of Crude Oil Quantitative Standard (Equal Mix by Weight Percent, Supelco) was used. The standard mixture contained 13 aliphatic hydrocarbons (C10-C18, C20, C22, $\mathrm{C} 24$ and $\mathrm{C} 28$ ) were analyzed in three concentration: $2.7 \mathrm{mg}, 4.0 \mathrm{mg}$ and $6.9 \mathrm{mg}$ of the mixture were dissolved in $1 \mathrm{~mL}$ of carbon disulfide. In diesel oil, 11 alkanes were determined (C11-C18, C20, C22, C24). The qualitative analysis of diesel oil samples was done comparing the retention time of standard hydrocarbons and oil components; the quantitative analysis was done using calibration method.

\section{Chemometric calculations}

ANOVA is a method used to assess effects of the categorical factors and their interactions (Lindman, 1991). Actually the model contained the following terms:

$$
\begin{aligned}
& Y=I n t e r c e p t+I+I 2+I 3+I * I 2+I * I 3+ \\
& I 2 * I 3+I * I 2 * I 3
\end{aligned}
$$

where $Y$ stands for the alkane concentration, $I$ is the type of bacterium (3 levels), $I 2$ is the indicator for emulsifier ( 1 = present, $2=$ not), $I 3$ is the type of yeast ( 4 levels). When the effect on different alkanes were treated separately, there were no multiple $y$ values (as average values were used), thus statistical significance of effects were not tested, only the visual analysis of effects was used for inference. In case of looking the effects on groups of alkanes the several alkanes play the role of repetitions, thus significance was tested as well.

\section{Sum of ranking differences (SRD) and its validation}

The novel ordering method has been described earlier by Héberger (2010) and its validation published thereafter Héberger and Kollár-Hunek (2011). SRD ordering is based on the comparisons of rank numbers. The rank numbers of the actual and a reference (benchmark) orderings are always compared (rank numbers are subtracted and their absolute values are built and added together for each systems). Such is the way all systems are compared (A1, $\mathrm{A} 2, \ldots, \mathrm{PE} 4)$ receives an SRD value. The smaller the SRD value, the "better" i.e. the less discrepancy can be observed, as compared to the reference ranking. The ordering is given by 11 alkanes C11-C18, C20, C22 and C24 (objects). In Figures 1-4 the results for only six alkanes (C12-C15, C18, C20 and C24) are presented as the most characteristic. The relationships concerned all 11 compounds are given in the text. Generally the averages of all methods (in this case, concentrations for all systems) are selected as a benchmark. However, such references rank the systems to the average, whereas the best system was that which provides the smallest concentration for all alkanes. The minimum concentration could be taken as an alternative to be selected for all systems, but no ranking can be made for 11 alkanes, as the minimum concentration is always zero (with one exception). A viable alternative is to select a very good system as governor for ranking. ANOVA results provide such a system: AE1 was selected for reference ranking (see later).

\section{Results and Discussion}

\section{Treating alkanes separately}

The results clearly indicate that the kind of bacterial and yeast strains in consortia have an influence on the biodegradation of the saturated aliphatic fractions. Biodegradation data were evaluated by using the ANOVA/GLM procedure. It allowed showing the influence of the different factors biodegradation efficiency. As the average of repeated experiments was used in ANOVA calculations, statistical significance of effects have not been tested.

The microbial consortiums are effective in hydrocarbon biodegradation; however, very important is the microbial composition. In the experiments twelve different consortia were used. The best results of biodegradation were observed for consortia with A. hydrophila KR4 (Figures 1-4).

The most characteristic figures are shown; the effects of all three factors can easily be seen.

Significant decrease of all aliphatic fraction content was observed after 7 days of experiments. Especially the consortia with C. maltosa EH60 - 2 and Y. lipolytica EH465 - 4 were effective. As not all consortia are satisfactorily effective in biodegradation of all fractions of diesel oil these two yeasts seem to be quite useful as they cause $\sim 100 \%$ degradation of the sample. This means that they could be used as diesel oil biodegradation in the environment.

Although a test for the significance of effects was not available, for the effect of the three factors (bacteria and yeast and presence or absence of emulsifier) and their interactions, Figures $1 \mathrm{a}$ and $1 \mathrm{~b}$ provide and easily show a perceivable overview of the degradation pattern. The differences between the lower molecular mass alkanes (Figure 1a) and the higher (Figure 1b) was also revealed.

The highest level of hydrocarbon biodegradation (in the system without biosurfactant) was also observed for two other consortia of $S$. maltophila KR7 with C. maltosa EH60 (X2) and Y. lypolitca EH465 (X4). The activity of these sys- 

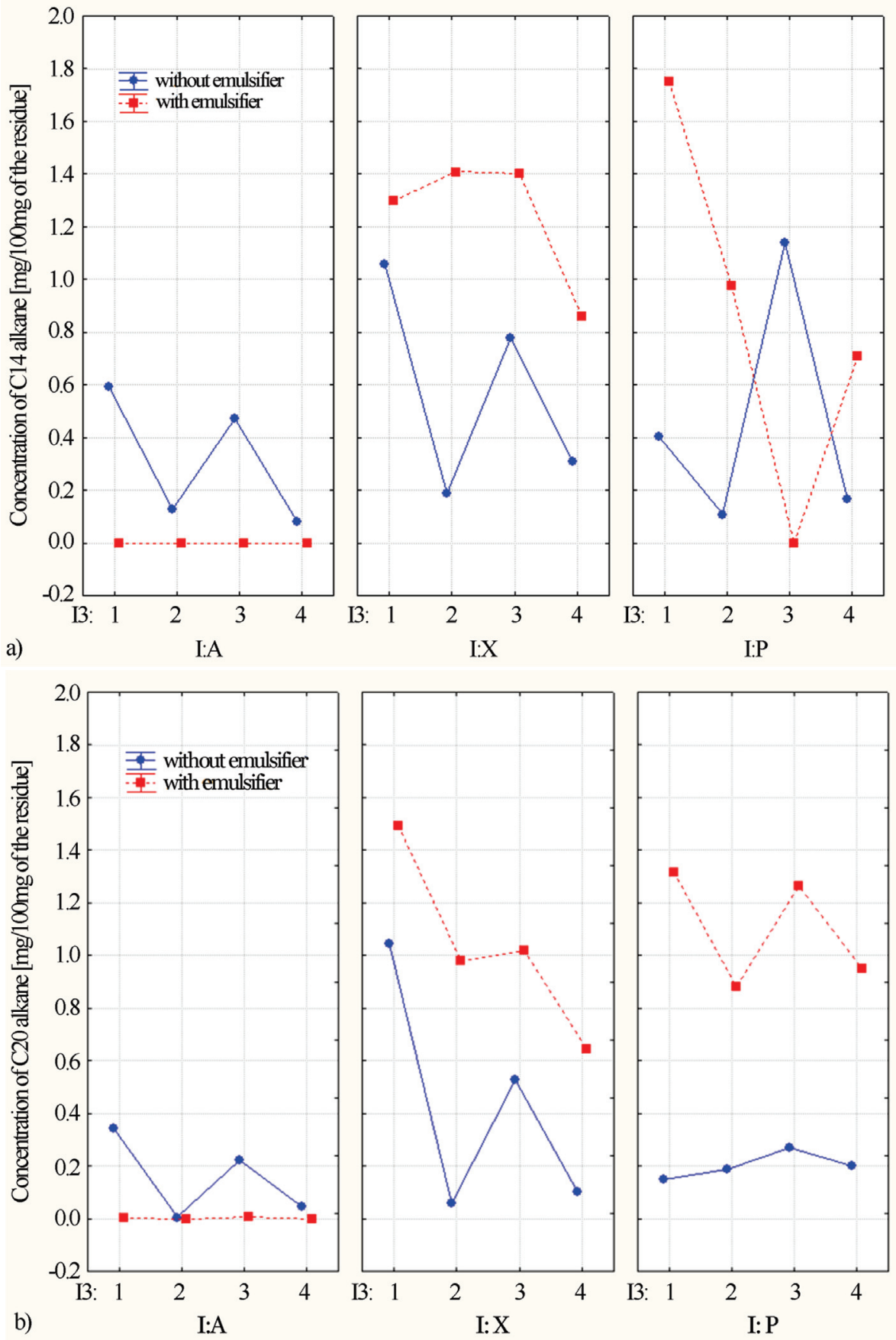

Figure 1 - Biodegradation of: a) C14 alkane and b) C20 alkane fraction from diesel oil; yeast strains: Candida maltosa EH15 - 1, EH60 - 2, Yarrowia lipolytica EH59 - 3, EH425 - 4; bacterial strains: Aeromonas hydrophila KR4 (A), Stenotrophomonas maltophila KR7 (X) Pseudomonas stutzeri KR7 (P).

tems is comparable with those of $A$. hydrophila KR4. A positive effect of the addition of C. maltosa EH60 was observed in its consortium with P. stutzeri KR7 (P2 and P4). These consortia were found to be the most effective biological systems in hydrocarbon biodegradation in comparison to other systems: yeasts strains - P. stutzeri KR7 (P2).

The addition of rhamnolipids biosurfactant to the system had a positive influence on hydrocarbon biodegra- dation in all four consortia A. hydrophila KR4 with yeast strains (AE1, AE2, AE3, AE4). In the case of S. maltophila KR7, the addition of rhamnolipids (XE1-XE4) did not have any positive effect on hydrocarbon biodegradation (Figure 2a,b). The biodegradation of aliphatic hydrocarbon fractions was significantly lower, than in the system without rhamnolipids. In these cases, the addition of biosurfactants to the bioremediation process was not a good solution. 
However, a different influence of rhamnolipids on hydrocarbon biodegradation was observed for the consortia P. stutzeri KR7 and Y. lypolitica EH56 (PE3). Fractions from $\mathrm{C} 11$ to $\mathrm{C} 15$ and from $\mathrm{C} 24$ to $\mathrm{C} 28$ were first biodegraded after the addition of rhamnolipids. Moreover, the greatest increase in the biodegradation of fractions from C17 to $\mathrm{C} 20$ was observed in the system without biosurfactants (Figure 3a,b).
Nikakhtari et al. (2009), observed a larger decrease in the amount of lighter compounds when biodegradation was carried out using a bacterial consortium. Gallego et al. (2001) demonstrated, that lighter hydrocarbons from diesel oil were degraded at a slower rate than heavier ones over 20 days. This was caused by increase of the amount of lighter hydrocarbons as a consequence of degradation of the heavier ones. Linear alkanes were degraded first and with
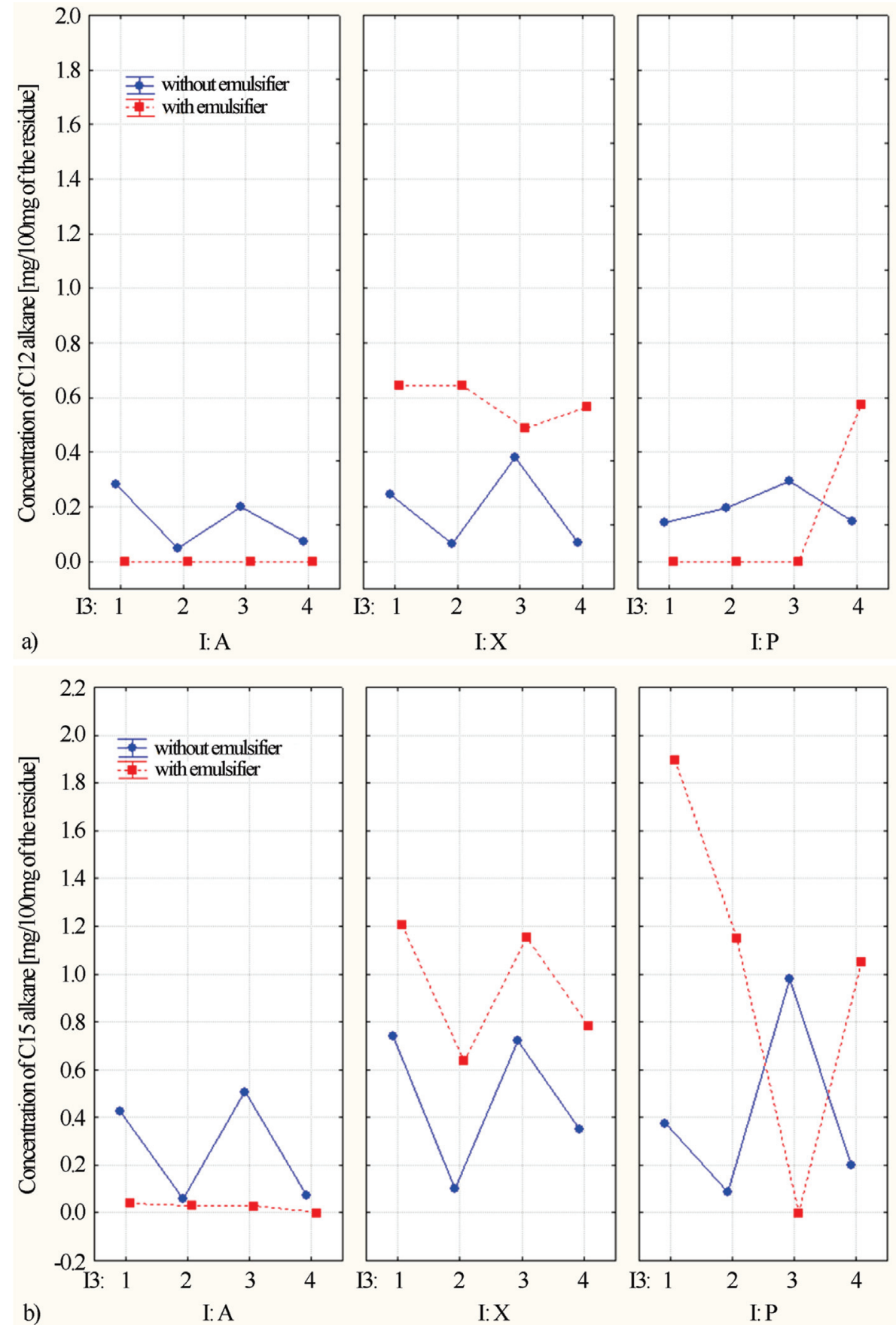

Figure 2 - Biodegradation of: a) C12 alkane and b) C15 alkane fraction from diesel oil; yeast strains: Candida maltosa EH15 - 1, EH60 - 2, Yarrowia lipolytica EH59 - 3, EH425 - 4; bacterial strains: Aeromonas hydrophila KR4 (A), Stenotrophomonas maltophila KR7 (X) Pseudomonas stutzeri KR7 (P). 

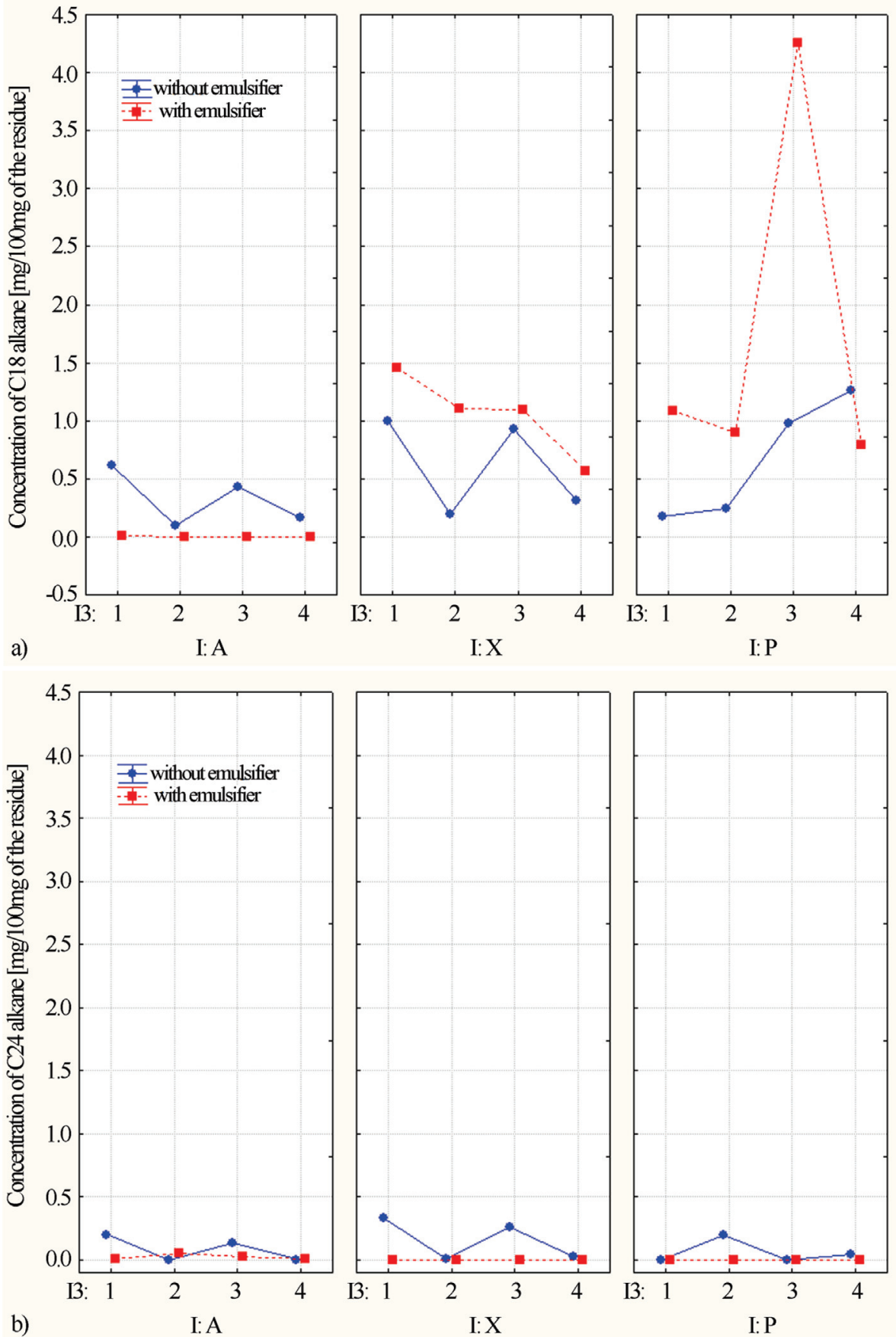

Figure 3 - Biodegradation of: a) C18 alkane and b) C24 alkane fraction from diesel oil; yeast strains: Candida maltosa EH15 - 1, EH60 - 2, Yarrowia lipolytica EH59 - 3, EH425 - 4; bacterial strains: Aeromonas hydrophila KR4 (A), Stenotrophomonas maltophila KR7 (X) Pseudomonas stutzeri KR7 (P)

higher yields. Moreover, chain length of the alkanes is important during their biodegradation, independently on initial rate of pollution in the range investigated by Seklemova et al. (2001).

\section{Treating alkanes in two groups (lighter and heavier alkanes)}

It is tempting to treat the data obtained for the different alkanes together. Lower and higher molecular mass al- kanes have been handled separately: C11-C20 and C22, $\mathrm{C} 24$, respectively. The data did not come from repeated experiments, but treating them as if they were - offers a possibility for significance testing. The difference in results (variation) shed light to the difference in behavior of different alkanes.

Treating data on C11-C20 together (lighter alkanes): the main effects of all factors are significant; from among the interactions only yeasts and emulsifier is significant 
(data not shown). The relatively large confidence ranges (e.g. at $I=\mathrm{P}, I 2=2, I 3=3$ combination) mean that the different alkanes behave in a different way.

The plot of (weighted) means with the full model (Eq. (1)) gives very similar conclusion as reached with alkanes separately for the two groups; lighter and heavy alkanes (Figures 4a,b).
The remaining hydrocarbon concentration after treatment was virtually zero when A. hydrophila KR4 is used together with emulsifier at all levels of yeasts factor. It is interesting to note that the use of two other bacteria without emulsifier is more advantageous. The relatively large confidence ranges (e.g. PE3) mean that the different alkanes (within the C11-C20 group) behave differently.
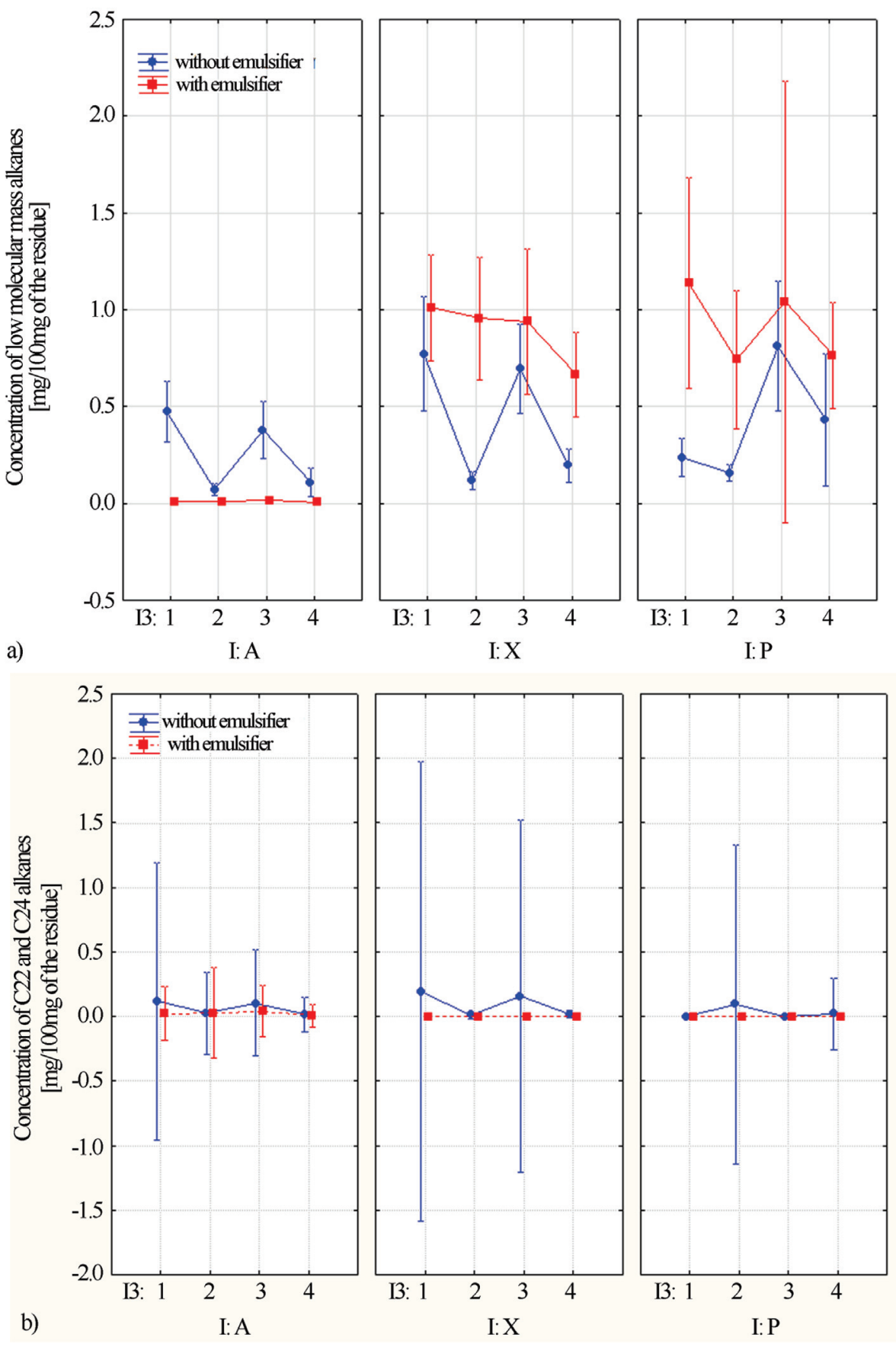

Figure 4 - Plot of weighted means a) lower molecular mass alkanes and b) higher molecular mass alkanes, the yeast strains: Candida maltosa EH15 - 1, EH60 - 2, Yarrowia lipolytica EH59 - 3, EH425 - 4; bacterial strains: Aeromonas hydrophila KR4 (A), Stenotrophomonas maltophila KR7 (X) Pseudomonas stutzeri KR7 (P). Error bars represent confidence interval (95\%). 
ANOVA table for the heavier hydrocarbon group is given as Table 1. The main effects of factor I, I2 and I3 are significant, from among the interactions only $\mathrm{I} 2 * \mathrm{I} 3$ is significant.

Treating data on C24 and C28 together (heavier alkanes): It is worth remarking that the formal significance test was based on a smaller amount of data (two alkanes). Thus, it is more difficult to reach significance here, as compared with the $\mathrm{C} 11-\mathrm{C} 20$ set.

The usage of emulsifier is more advantageous, especially when combined with bacteria denoted by $X$ and $P$. The concentration of hydrocarbons after treatment is convincingly zero. The bacterial strain denoted by $A$ also gives zero in average with emulsifier, but there is a more emphasized difference between different compounds. More varying results could be observed without emulsifier.

ANOVA table for the heavier hydrocarbon group is given as Table 2. Only the intercept and the I2 are significant. The formal significance test is based on smaller amount of data, thus it is more difficult to reach significance here, as compared with the C11-C20 set.

Table 1 - Univariate tests of significance for C11 -C20 hydrocarbons degradation (Over-parameterized model, Type III decomposition).

\begin{tabular}{lccccc}
\hline & $\begin{array}{c}\text { Sum of } \\
\text { squares }\end{array}$ & $\begin{array}{c}\text { Degree of } \\
\text { freedom }\end{array}$ & $\begin{array}{c}\text { Mean } \\
\text { squares }\end{array}$ & $\mathrm{F}$ & $\mathrm{p}$ \\
\hline Intercept & $\mathbf{5 1 . 7 7 7 0 1}$ & $\mathbf{1}$ & $\mathbf{5 1 . 7 7 7 0 1}$ & $\mathbf{2 8 2 . 2 6 3 3}$ & $\mathbf{0 . 0 0 0 0 0 0}$ \\
I & $\mathbf{1 3 . 6 6 1 0 8}$ & $\mathbf{2}$ & $\mathbf{6 . 8 3 0 5 4}$ & $\mathbf{3 7 . 2 3 6 8}$ & $\mathbf{0 . 0 0 0 0 0 0}$ \\
I2 & $\mathbf{3 . 0 5 3 8 3}$ & $\mathbf{1}$ & $\mathbf{3 . 0 5 3 8 3}$ & $\mathbf{1 6 . 6 4 8 0}$ & $\mathbf{0 . 0 0 0 0 6 6}$ \\
I3 & $\mathbf{4 . 1 1 7 0 1}$ & $\mathbf{3}$ & $\mathbf{1 . 3 7 2 3 4}$ & $\mathbf{7 . 4 8 1 3}$ & $\mathbf{0 . 0 0 0 0 9 2}$ \\
I*I2 & $\mathbf{6 . 3 7 9 8 4}$ & $\mathbf{2}$ & $\mathbf{3 . 1 8 9 9 2}$ & $\mathbf{1 7 . 3 8 9 9}$ & $\mathbf{0 . 0 0 0 0 0 0}$ \\
I*I3 & 1.19243 & 6 & 0.19874 & 1.0834 & 0.373768 \\
I2*I3 & 1.17477 & 3 & 0.39159 & 2.1348 & 0.097204 \\
I*I2* I 3 & 1.61646 & 6 & 0.26941 & 1.4687 & 0.190859 \\
Error & 35.21956 & 192 & 0.18344 & & \\
\hline
\end{tabular}

Significant values (at the $5 \%$ level) are indicated by bold.

Table 2 - Univariate tests of significance for $\mathrm{C} 24$ and C28 hydrocarbons degradation (Over-parameterized model, Type III decomposition).

\begin{tabular}{lccccc}
\hline & $\begin{array}{c}\text { Sum of } \\
\text { squares }\end{array}$ & $\begin{array}{c}\text { Degree of } \\
\text { freedom }\end{array}$ & $\begin{array}{c}\text { Mean } \\
\text { squares }\end{array}$ & $\mathrm{F}$ & $\mathrm{p}$ \\
\hline Intercept & $\mathbf{0 . 0 6 2 3 6 4}$ & $\mathbf{1}$ & $\mathbf{0 . 0 6 2 3 6 4}$ & $\mathbf{1 4 . 6 2 4 1 9}$ & $\mathbf{0 . 0 0 0 8 2 1}$ \\
I & 0.010823 & 2 & 0.005412 & 1.26898 & 0.299313 \\
I2 & $\mathbf{0 . 0 3 7 1 8 5}$ & $\mathbf{1}$ & $\mathbf{0 . 0 3 7 1 8 5}$ & $\mathbf{8 . 7 1 9 6 2}$ & $\mathbf{0 . 0 0 6 9 3 9}$ \\
I3 & 0.015997 & 3 & 0.005332 & 1.25040 & 0.313582 \\
I*I2 & 0.010107 & 2 & 0.005053 & 1.18499 & 0.323011 \\
I*I3 & 0.028517 & 6 & 0.004753 & 1.11451 & 0.383278 \\
I2*I3 & 0.011710 & 3 & 0.003903 & 0.91528 & 0.448349 \\
I*I2*I3 & 0.027435 & 6 & 0.004573 & 1.07224 & 0.406258 \\
Error & 0.102347 & 24 & 0.004264 & & \\
\hline
\end{tabular}

\section{Results of SRD ranking}

Naturally, one cannot expect exactly the same evaluation and results from the ranking by SRD as earlier by ANOVA, because the pattern is contradictory: higher molecular mass alkanes behave differently. However, the general picture is supported by SRD calculations (Figure 5).

Accepting the best consortium denoted by AE1 $(A$. hydrophila KR4 (A), C. maltosa EH15 - 1 using surfactant) five systems can be classified as acceptable AE1 (reference), $\mathrm{AE} 3, \mathrm{AE} 2, \mathrm{AE} 4$ and $\mathrm{PE} 3$ supporting the general conclusion of positive effect of emulsifiers.

Many consortia provide no better ranking as the random numbers do. It is interesting that one consortium is located above the upper significance level denoted by XE2 (S. maltophila KR7, C. maltosa EH60 - 2 with emulsifiers). This unorthodox behavior is rare; it means a reverse ordering for XE2 than AE1.

Diverse influence of the rhamnolipids might be a result of their use as a coal and energy source by microorganisms present in the consortium. Bacterial and yeast strains used in experiments grew on rhamnolipids as a carbon source (unpublished data). No significant increase in biomass was observed after addition of rhamnolipids to bacterial strains in comparison to a blank sample. The only exception was found for A. hydrophila KR4, where in increase in biomass was close to that found in the blank sample. One should note, that in the blank sample the growth of microorganisms resulted from the consumption of glucose as a carbon source. Glucose is a simple carbon source, which is easily metabolized by a microorganism. Surfactants could increase the bioavailability of water insoluble compounds. However, a more promising solution in hydrocarbon biodegradation may be the use of biosurfactants. These compounds have more advantages over synthetic surfactants (Makkar and Rockne, 2003). Biosurfactants are less toxic for the environment and are readily biodegradable. The effect of surfactants on the biodegradation of hydrocarbons is the theme of many researchers (Banat et al., 2010; Bordoloi and Konwar, 2009; Das et al., 2008). Zhang and Miller (1994) observed that the addition

\section{CRRN results (Discrete, $\mathrm{n}=11$ )}

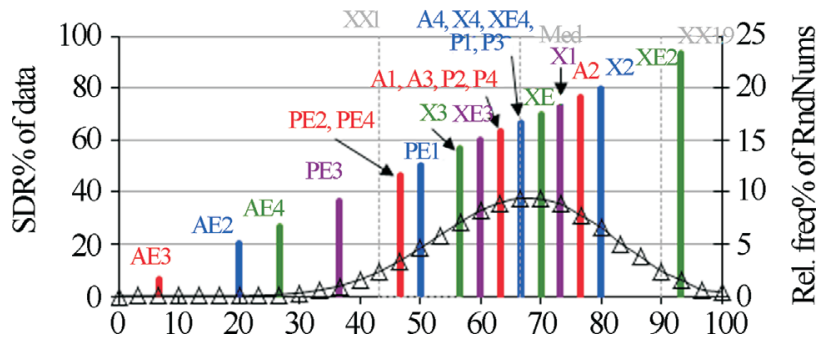

Figure 5 - Sum of absolute ranking differences values (scaled between 0 and 100) for consortia (reference was AE1) are plotted on X and Y-left axes alike. the top of the lines represent a line of $45^{\circ}$ The information is carried by the proximity (and/or distance) of lines. Y-right axis shows the relative frequencies for validation by random numbers (Gauss-like curve). 
of rhamnolipids to a biodegradation mixture of slightly soluble organic compounds in some cases led to enhanced biodegradation, while in other cases it inhibited the process. Rahman et al. (2003) observed that rhamnolipids are effective as an enhancer in biodegradation of alkane in petroleum sludge. However, Mata-Sandoval et al. (2001) described that increasing solubilization of hydrophobic organic compounds after addition of rhamnolipids does not guarantee enhanced biodegradation. The effectiveness of surfactants on hydrocarbon biodegradation depends on different factors, e.g. interactions between biosurfactants and hydrocarbon or between biosurfactants and microorganisms (Haritash and Kaushik, 2009). Biosurfactants could affect hydrocarbon biodegradation through cell surface modification (Kaczorek and Olszanowski, 2011; Obuekwe et al., 2009). The striking novelty of the present investigation is obvious from the analysis of the diversity of biodegradation of different diesel fractions. It has also been shown that the influence of rhamnolipids depends on the nature and composition of the microbiological consortium.

\section{Conclusions}

Results of gas chromatographic analysis supported by chemometric techniques enabled the assessment of the usefulness of examined biological agents. Several consortia consisting of selected bacteria and yeasts strains, as well as rhamnolipids proved to be sufficient for diesel oil elimination from the environment. The effect of the addition of rhamnolipids was found to be positive in some cases (consortia of A. hydrophila KR4 with C. maltosa and $Y$. lipolytica yeasts). However, the negative influence on consortia bioactivity was also observed. A ranking procedure based on the sum of ranking differences supports the unambiguous positive effect of an emulsifier.

\section{Acknowledgments}

This work was supported by Grant No. N N304 163337, Polish Ministry of Science and Higher Education, as well as the Polish-Hungarian exchange program of the Polish and Hungarian Academies of Sciences for 20112013.

\section{References}

Abdel-Mawgoud AM, Lepine F, Deziel E (2010) Rhamnolipids: diversity of structures, microbial origins and roles. Appl Microbiol Biotechnol 86:1323-1336.

Aronstein BN, Alexander M (1993) Effect of a nonionic surfactant added to the soil surface on the biodegradation of aromatic hydrocarbons within the soil. Appl Microbiol Biotechnol 39:386-390.

Banat IM, Franzetti A, Gandolfi I, Bestetti G, Martinotti MG, Fracchia L; Smyth TJ, Marchant R (2010) Microbial biosurfactants production, applications and future potential. Appl Environ Microbiol 87:427-444.
Benincasa M, Abalos A, Oliveira I, Manresa A (2004) Chemical structure, surface properties and biological activities of the biosurfactant produced by Pseudomonas aeruginosa LBI from soap-stock. Anton Leeuw Int JG 85:1-8.

Bordoloi NK, Konwar BK (2009) Bacterial biosurfactants in enhancing solubility and metabolism of petroleum hydrocarbons. J Hazard Mater 170:495-505.

Chrzanowski L, Bielicka-Daszkiewicz K, Owsianiak M, Aurich A, Kaczorek E, Olszanowski A (2008) Phenol and n -alkanes (C12 and C16) utilization: influence on yeast cell surface hydrophobicity. World J Microbiol Biotechnol 24:1943-1949.

Das P, Mukherjee S, Sen R (2008) Improved bioavailability and biodegradation of a model polyaromatic hydrocarbon by a biosurfactant producing bacterium of marine orgin. Chemosphere 72:1229-1234.

Gallego JLR, Loredo J, Llamas JF, Vazguez F Sanchez J (2001) Bioremediation of diesel-contaminated soils: Evaluation of potential in situ techniques by study of bacterial degradation. Biodegradation 12:325-335.

Haba E, Pinazo A, Jauregui O, Espuny MJ, Infante MR, Manresa A (2003). Physicochemical characterization and antimicrobial properties of rhamnolipids produced by Pseudomonas aeruginosa 47T2 NCBIM 40044. Biotechnol Bioeng 81:316-322

Haritash AK, Kaushik CP (2009) Biodegradation aspects of polycyclic aromatic hydrocarbons (PAHs): a review. J Hazard Mater 169:1-15.

Héberger K (2010) Sum of ranking differences compares methods or models fairly. Trends Anal Chem 29:101-109.

Héberger K, Kollár-Hunek K (2011) Sum of ranking differences for method discrimination and its validation: comparison of ranks with random numbers. J Chemometr 25:151-158.

Horakova DMV, Nemec M (2000). RC1 Consortium for soil decontamination: Its preparation and use. Remediation engineering of contaminated soils. In: Wise, D.L., Tranto, D.J., Cichon, E.J., Injang, H.I., Stottmeister, U. (eds). Remediation engineering of contaminated soils. Marcel Dekker Inc, New York, pp 357-372.

Kaczorek E, Olszanowski A (2011) Uptake of hydrocarbon by Pseudomonas fluorescens (P1) and Pseudomonas putida (K1) strains in the presence of surfactants: A Cell surface modification. Water Air Soil Poll 214:451-459.

Leahy JG, Colwell RR (1990) Microbial degradation of hydrocarbons in the environment. Microbiol Rev 54:305-315.

Lindman HR (1991) Analysis of Variance in Experimental Design. Springer Verlag, New York.

Liu Z, Jacobson AM, Luthy RG (1995) Biodegradation of naphthalene in aqueous nonionic surfactant system. Appl Environ Microbiol 61:145-151.

Makkar RS, Rockne KJ (2003) Comparison of synthetic surfactants and biosurfactants in enhancing biodegradation of polycyclic aromatic hydrocarbons. Environ Toxicol Chem 22:2280-2292.

Mata-Sandoval JC, Karns J, Torrents A (2001) Influence of rhamnolipids and Triton X-100 on the biodegradation of three pesticides in aqueous phase and soil slurries. J Agr Food Chem 49:3296-3303.

Nikakhtari H, Pardeep K, Nemati M, Hill GA (2009) Physical and biological treatment of oil-contaminated soil in a baffled roller bioreactor. Bioremed J 13:130-140. 
Obuekwe CO, Al-Jadi ZK, Al-Saleh ES (2009) Hydrocarbon degradation in relation to cell-surface hydrophobicity among bacterial hydrocarbon degraders from petroleum-contaminated Kuwait desert environment. Int Biodeter Biodegr 63:273-279.

Palittapongarnpim M, Pokethitiyook P, Upatham E.S, Tangbanluekal L (1998) Biodegradation of crude oil by soil microorganisms in the tropic. Biodegradation 9:83-90.

Rahman KSM, Rahman TJ, Kourkoutas Y, Petsas I, Marchant R, Banat IM (2003) Enhanced bioremediation of n-alkane in petroleum sludge using bacterial consortium amended with rhamnolipid and micronutrients. Bioresource Technol 90:159-168.
Seklemova E, Pavlova A, Kovacheva K (2001) Biostimulationbased bioremediation of diesel fuel: field demonstration. Biodegradation 12:311-316.

Volkering F, Breurke AM, van Andel JG, Rulkens WH (1995) Influence of nonionic surfactants on bioavailability and biodegradation of polycyclic aromatic hydrocarbons. Appl Environ Microbiol 61:1699-1705.

Zhang Y, Miller RM (1994) Effect of a Pseudomonas rhamnolipid biosurfactant on cell hydrophobicity and biodegradation of octadecane. Appl Environ Microbiol 60, 2101-2106.

All the content of the journal, except where otherwise noted, is licensed under a Creative Commons License CC BY-NC. 